\title{
Burden of migraine: international perspectives
}

\author{
M. Leonardi · A. Raggi
}

(C) Springer-Verlag Italia 2013

\begin{abstract}
The newly released version of the Global Burden of Disease (GBD 2010) ranks migraine between the most disabling diseases, the first among neurological ones, with a two-fold increase with respect to the previous GBD 2000 version. Almost $3 \%$ of worldwide disability attributable to a specific disease, in terms of years lived with a disability, is due to migraine. The public health impact of migraine and headache disorders is thus a consolidated fact; however, further research is needed to provide stronger recognition of and policy actions for headache disorders in general, and in particular with regard to chronic forms of headache.
\end{abstract}

Keywords Migraine - Headaches - Disability ·

Burden of disease $\cdot$ YLL $\cdot$ DALYs

\section{Introduction}

Migraine is one of the most common diseases, with lifetime prevalence ranging between 14 and $16 \%[1,2]$ : it is commonly associated to high costs, in particular with regard to indirect costs, i.e. those associated to reduced productivity [3]. Although of relevance, this view is partial as it does not really capture the reduction in health due to migraine.

Health loss and disability are instead the core concepts of the Global Burden of Disease (GBD) study, whose last

M. Leonardi ( $₫)$. A. Raggi

Neurology Public Health and Disability Unit,

Neurological Institute C. Besta IRCCS Foundation,

Via Celoria 11, 20133 Milan, Italy

e-mail: leonardi@istituto-besta.it update was released in December 2012: it enables to compare diseases and disease-related risk factors in different places around the world and to understand what are the most important contributors to health loss and to disability [4]. GBD is based on the summary measure of health called disability-adjusted life years (DALYs): these are based on an evaluation of the combination of years of life lost (YLLs) and years lived with a disability (YLDs). YLDs have been computed as the prevalence of each disease multiplied by the disability weights calculated for each disease and adjusted for relevant comorbidities [5, 6]. Aim of this study is to synthetically present the figures related to migraine referred to the last 20 years, with reference to the data for 1990 [7], 2000 [8], 2004 [9] and to the recently released data for $2010[5,10]$. Data have also been directly taken from databases available on WHO's website (accessed 12 February 2013) at http://www.who. int/healthinfo/global_burden_disease/estimates_regional/en/ index.html.

\section{The burden associated to migraine}

One of the peculiarities of burden associated to migraine is that it is only made of YLDs, as there is no mortality associate to it. Table 1 reports figures for DALYs and YLDs between 1990 and 2010, showing a consistent increase in the last 6 years. What is particularly interesting to note is that the most recent reports state that migraine alone is responsible of almost $3 \%$ of disability attributable to a specific disease worldwide, also in consideration of its comorbidity. This places migraine as the eighth most burdensome diseases, the seventh among non-communicable diseases and the first among the neurological disorders ranked in the GBD report. 
Table 1 DALY and YLD associated to migraine for the period 1990-2010

\begin{tabular}{lllll}
\hline & 1990 & 2000 & 2004 & 2010 \\
\hline Total DALY & 15,927 & 7,539 & 7,765 & 22,362 \\
$\begin{array}{l}\text { \% over total DALYs } \\
\text { for all diseases }\end{array}$ & 0.64 & 0.51 & 0.51 & 0.89 \\
$\quad \begin{array}{l}\text { Total YLD } \\
\text { \% over total DALYs }\end{array}$ & 15,927 & 7,539 & 7,765 & 22,362 \\
$\quad$ for all diseases & & 1.4 & 1.3 & 2.9 \\
DALY-YLD/100,000 & 300 & 125 & 121 & 325 \\
\hline
\end{tabular}

$D A L Y$ disability-adjusted life years, $Y L D$ years lived with a disability

\section{Discussion}

The new GBD reports support a clear fact: migraine is one of the leading causes of disability worldwide, as it has high prevalence and it is associated to several other prevalent and disabling conditions. Among them, anxiety and affective disorders are the most common [11], but there are reports for comorbidity to stroke, cardiovascular diseases, epilepsy, respiratory and gastro-intestinal disturbances [12]. It is probably due to the recognition of such comorbidities that the disability weight associated to migraine was reported as 0.433 [6]: this means that during a migraine attack, a person with migraine has $43.3 \%$ of disability on a $0-100 \%$ scale, comparable to the first 2 days after acute myocardial infarction (0.422), severe dementia $(0.438)$ or untreated spinal cord injury below the neck $(0.440)$.

The new GBD is clearly a step forward in the recognition of the burden associated to migraine and headache disorders in general and much of this advancement is probably due to the efforts by the international "Lifting the Burden" campaign towards worldwide data collection on migraine [13]. However, we share the concern recently raised by Steiner and colleagues [14]: the exclusion of medication overuse headache is critical, as it adds enormously to the burden associated to headache disorders. So, in our opinion, the criticism of GBD 2010 must be taken as an opportunity: further studies must be undertaken that show the impact of all headache disorders using reliable disability outcome measures that enable comparative analysis across different headache disorders [15], as well as in different chronic conditions [16] and in different countries.
Conflict of interest I certify that there is no actual or potential conflict of interest in relation to this article.

\section{References}

1. Lj Stovner, Hagen K, Jensen R (2007) The global burden of headache: a documentation of headache prevalence and disability worldwide. Cephalalgia 27:193-210

2. Stovner LJ, Andree C (2010) Prevalence of headache in Europe: a review for the Eurolight project. J Headache Pain 11:289-299

3. Linde M, Gustavsson A, Stovner LJ et al (2012) The cost of headache disorders in Europe: the Eurolight project. Eur J Neurol 19:703-711

4. Murray CJL, Ezzati M, Flaxman AD et al (2012) GBD 2010: design, definitions, and metrics. Lancet 380:2063-2066

5. Vos T, Flaxman AD, Naghavi M et al (2012) Years lived with disability (YLDs) for 1160 sequelae of 289 diseases and injuries 1990-2010: a systematic analysis for the Global Burden of Disease Study 2010. Lancet 380:2163-2196

6. Salomon JA, Vos T, Hogan DR (2010) Common values in assessing health outcomes from disease and injury: disability weights measurement study for the Global Burden of Disease Study. Lancet 380:2129-2143

7. Murray CJL, Lopez AD (eds) (1996) The global burden of disease: a comprehensive assessment of mortality and disability from diseases, injuries and risk factors in 1990 and projected to 2020. Harvard School of Public Health on behalf of the World Health Organization and the World Bank, Cambridge

8. WHO (2001) The world health report 2001—mental health: new understanding, new hope. WHO, Geneva

9. WHO (2008) The global burden of disease: 2004 update. WHO, Geneva

10. Murray CJL, Vos T, Lozano R et al (2012) Disability-adjusted life years (DALYs) for 291 diseases and injuries in 21 regions, 1990-2010: a systematic analysis for the Global Burden of Disease Study 2010. Lancet 380:2193-2223

11. Antonaci F, Nappi G, Galli F et al (2011) Migraine and psychiatric comorbidity: a review of clinical findings. J Headache Pain 12:115-125

12. Diener HC, Küper M, Kurth T (2008) Migraine-associated risks and comorbidity. J Neurol 255:1290-1301

13. Steiner TJ (2004) Lifting the burden: the global campaign against headache. Lancet Neurol 3:204-205

14. Steiner TJ, Stovner LJ, Birbeck GL (2013) Migraine: the seventh disabler. J Headache Pain 14:1

15. D'Amico D, Grazzi L, Usai S et al (2011) Disability in chronic daily headache: state of the art and future directions. Neurol Sci 32:S71-S76

16. Garin O, Ayuso-Mateos JL, Almansa J et al (2010) Validation of the "World Health Organization Disability Assessment Schedule, WHODAS-2" in patients with chronic diseases. Health Qual Life Outcomes 8:51 